\title{
Contribuições da religiosidade no enfrentamento da síndrome de burnout em profissionais de educação física
}

\author{
The possible contributions of religiosity in confronting the burnout syndrome in physical educacion professionals \\ Las posibles aportaciones de la religiosidad al afrontamiento del síndrome burnout en los professionales de educación \\ física
}

\begin{abstract}
RESUMO
Objetivo: Descrever as possíveis contribuições da religiosidade no enfrentamento da Síndrome de Burnout (SB) em profissionais de Educação Física. Método: Foi realizada uma revisão bibliográfica nas seguintes bases de dados: CAPES e SciELO, com publicações no período de 2010 a 2020. Utilizados como critérios de inclusão: Educação Física; Síndrome de Burnout; Transtornos Mentais e critérios de exclusão: artigos que abordavam outros temas; duplicados nas duas bases de dados; não atendiam o recorte temporal; publicados em outros idiomas. Resultados: A Síndrome de Burnout (SB) possui uma grande incidência de diagnósticos realizados na atualidade, segundo a literatura científica, devido à sobrecarga exigida dos profissionais da área de Educação Física. Conclusão: 0 envolvimento religioso apresenta resultado positivo ante a saúde mental, de modo que os futuros profissionais devem saber lidar com todas as exigências e responsabilidades da profissão, assim como saber equilibrar tais funções dentro da área de atuação do educador.
\end{abstract}

DESCRITORES: Educação Física; Síndrome de Burnout; Transtornos Mentais.

\section{ABSTRACT}

Objective: To describe the possible contributions of religiosity in coping with Burnout Syndrome (SB) in Physical Education professionals. Method: A bibliographic review was carried out in the following databases: CAPES and SciELO, with publications from 2010 to 2020. Used as inclusion criteria: Physical Education; Burnout syndrome; Mental Disorders and exclusion criteria: articles that addressed other topics; duplicated in the two databases; did not meet the time frame; published in other languages. Results: Burnout Syndrome (SB) has a high incidence of diagnoses performed today, according to scientific literature, it occurs due to the overload required of professionals in the area of Physical Education. Conclusion: Religious involvement shows positive results in relations to mental health, since future professionals must know how to deal with all the demands and responsibilities of the profession, as well as knowing how to balance these functions within the area of activity of the educator.

DESCRIPTORS: Physical Education; Burnout syndrome; Mental Disorders.

\section{RESUMEN}

Objetivo: Describir las posibles contribuciones de la religiosidad en el afrontamiento del Síndrome de Burnout (SB) en profesionales de la Educación Física. Método: Se realizó una revisión bibliográfica en las siguientes bases de datos: CAPES y SciELO, con publicaciones de 2010 a 2020. Usados como criterios de inclusión: Educación Física; Síndrome de burnout; Trastornos mentales y criterios de exclusión: artículos que abordaron otros temas; duplicado en las dos bases de datos; no cumplió con el marco de tiempo; publicado en otros idiomas. Resultados: El Síndrome de Burnout (SB) tiene una alta incidencia de diagnósticos realizados en la actualidad, según la literatura científica, se produce debido a la sobrecarga que se requiere de los profesionales en el área de la Educación Física. Conclusión: La participación religiosa muestra resultados positivos en relación con la salud mental, ya que los futuros profesionales deben saber atender todas las demandas y responsabilidades de la profesión, así como saber equilibrar estas funciones dentro del ámbito de actuación del educador.

DESCRIPTORES: Educación Física; Síndrome de Burnout; Desordenes mentales.

RECEBIDO EM: 25/08/2020 APROVADO EM: 23/10/2020 


\section{Rafael Ferreira Pureza de Oliveira}

Profissional de Educação Física, professor de Educação Física do Instituto Federal Fluminense (IFF), Campus Santo Antônio de Pádua, mestrando em Ciências das Religiões, Faculdade Unida de Vitória (FUV).

ORCID: 0000-0003-2959-4351

\section{Michele de Oliveira Pereira}

Pedagoga, técnica do programa saúde na escola do Município de Vila Velha - ES e professora nas séries iniciais do fundamental do Município de Cariacica - ES, mestranda em Ciência das Religiões, Faculdade Unida de Vitória (FUV).

ORCID: 0000-0002-8218-0900

\section{Elson dos Santos Gomes Junior}

Sociólogo, professor de Sociologia do Instituto Federal Fluminense (IFF), Campus Santo Antônio de Pádua, mestre em Sociologia Política pela Universidade Estadual do Norte Fluminense Darcy Ribeiro (UENF).

ORCID: 0000-0002-7222-8288

\section{Ronaldo Cardoso de Oliveira}

Sacerdote, psicanalista, mestrando em Ciências das Religiões, Faculdade Unida de Vitória (FUV).

ORCID: 0000-0002-5437-3879

\section{Alexsandro Monteiro Pontini}

Professor de Arte, mestrando em Ciências das Religiões, Faculdade Unida de Vitória (FUV).

ORCID: 0000-0003-0171-5473

\section{Kelly Ramos do Rosário}

Advogada, orientadora no núcleo de prática jurídica da Faculdade de Direito do Vale do Rio Doce (FADIVALE), mestranda em Ciência das Religiões, Faculdade Unida de Vitória (FUV).

ORCID: 0000-0001-7267-3229

\section{INTRODUÇÃO}

egundo a Classificação Brasileira de Ocupações (CBO), identifica-se que os profissionais de Educação Física são reconhecidos enquanto profissão da área da saúde, sob o código 2241-40. Dessa maneira, trata-se de uma ocupação que estrutura e realiza ações de promoção da saúde por intermédio das atividades físicas e de lazer, exercícios físicos e práticas corporais na prevenção primária, secundária e terciária no SUS ${ }^{(1)}$.

No que concerne aos profissionais de Educação Física, percebe-se que o estresse e o over training representam termos que são confundidos no ambiente de trabalho destes profissionais ${ }^{(2)}$. Entretanto, verifica-se que o conceito mais recente de estresse parte do princípio da experiência de emoções negativas desagradáveis, como raiva, frustração, ansiedade, depressão e nervosismo, resultantes de algum aspecto do trabalho. Diante disso, a exaustão foi conceituada como uma condição psicológica que leva à despersonalização, dimi- nuição da capacidade de rendimento e da autoestima do trabalhador ${ }^{(3)}$.

Desse modo, a manutenção de elevados níveis de estresse pode acarretar no surgimento de problemas de saúde, dentre os quais a Síndrome de Burnout (SB). Assim, no Brasil, a Síndrome de Burnout (SB) é igualmente conhecida como a Síndrome do Esgotamento Profissional e na tabela de transtornos mentais e do comportamento está classificada com o CID-10 código Z 73.0 (2).

Recentemente, a Síndrome de Burnout foi incluída na $11^{\text {a }}$ Revisão da Classificação Internacional de Doenças como um fenômeno ocupacional, resultante de estresse crônico no local de trabalho que não foi gerenciado com sucesso. É uma doença relacionada aos mecanismos estressores do meio laboral e fundamenta-se em uma perspectiva sociopsicológica que se relaciona com a fase final e processo contínuo de estresse ${ }^{(4)}$.

A Síndrome de Burnout e o estresse relacionado ao trabalho devem ser considerados como duas construções diferentes, uma vez que, representam dois fatores de risco psicossocial diferentes, que devem ser abordados especificamente pelos empregadores no local de trabalho ${ }^{(5)}$.

O sofrimento ocupacional não é considerado uma doença, mas, se excessivo e prolongado, pode resultar em inúmeras doenças tanto físicas quanto mentais, incluindo a SB. Logo, também pode ser considerada uma consequência do estresse ocupacional de longo prazo associado a resultados negativos de saúde nos níveis individual e organizacional, incluindo problemas de ordem mental como ansiedade e depressão, baixa satisfação no trabalho, baixo desempenho e alto absentismo e taxas de rotatividade ${ }^{(6)}$.

No que tange à religiosidade, é entendida enquanto a manifestação do sagrado, de modo que a justaposição ao transcendente relacionado às escolhas da fé estabelece princípios que compõem o indivíduo, família e sociedade. Além disso, é percebido que a religiosidade se desponta como um importante elemento de proteção contra o surgimento da SB, favorecendo a promo- 
ção da qualidade de vida nos profissionais de Educação Física ${ }^{(6,7)}$.

Portanto, com base nos argumentos expostos, identifica-se a premência de uma maior atenção em relação à saúde mental destes profissionais. O problema de pesquisa proposto nesse estudo foi: De que maneira a religiosidade pode contribuir no enfrentamento da Síndrome de Burnout em profissionais de Educação Física? De tal modo, o presente estudo tem por objetivo descrever as possíveis contribuições da religiosidade no enfrentamento da SB em profissionais de Educação Física.

\section{MÉTODO}

Para realizar a presente pesquisa, foi feita uma revisão de literatura, a fim de encontrar pesquisas científicas publicadas que versam sobre o tema da religiosidade como enfrentamento da Síndrome de Burnout, principalmente em profissionais de Educação Física. A revisão de literatura se vale de artigos e estudos publicados, utilizando critérios de inclusão e exclusão a fim de selecionar os estudos viáveis para a pesquisa. $\mathrm{O}$ presente estudo contou com uma consulta avançada nas bases de dados de periódicos da Coordenação de Aperfeiçoamento de Pessoal de Nível Superior (CAPES) e na Scientific Eletronic Library On-Line (SciELO), utilizando-se dos seguintes descritores: Educação Física, Síndrome de Burnout e Transtornos Mentais. Foram utilizados artigos publicados em língua portuguesa, a fim de revisar o

Figura 1- Fluxograma do percurso metodológico da busca, no período de 2010 até o presente momento.
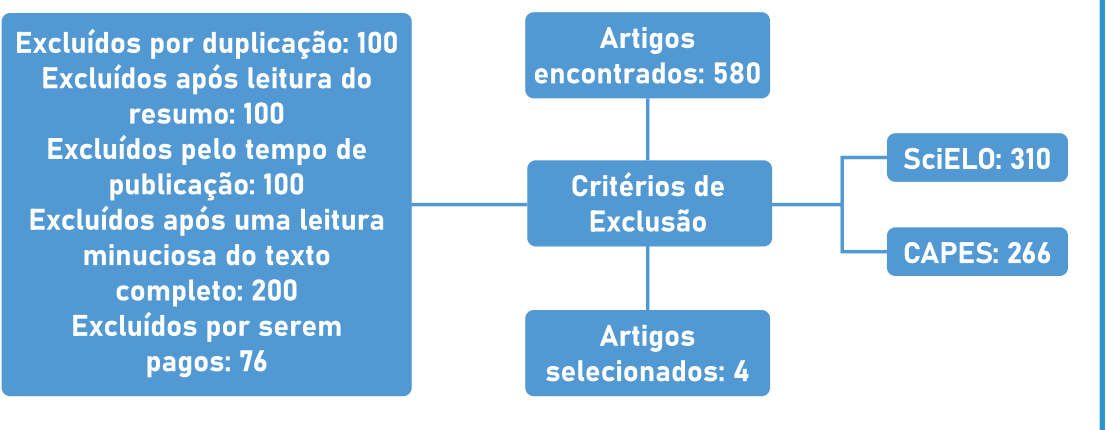

Para realizar a presente pesquisa, foi feita uma revisão de literatura, a fim de encontrar pesquisas que versam sobre o tema da religiosidade como enfrentamento da Síndrome de Burnout, principalmente em profissionais de Educação Física. científicas publicadas acervo nacional acerca do tema. Para o cruzamento dos descritores foi utilizado o operador booleano "AND". A pesquisa foi feita entre 04 de maio e 01 de junho de 2020, e contou com estudos publicados no período de 2010 a 2020.

Foram encontrados um total de 580 artigos, os quais foram analisados conforme os critérios de inclusão e exclusão. Para critério de inclusão foram utilizados estudos que abordavam as temáticas da Educação Física aliadas à Síndrome de Burnout e Transtornos Mentais, publicados entre 2010 e 2020, em língua portuguesa; e como critério de exclusão foram retirados da amostragem artigos que abordavam outros temas, artigos duplicados nas duas bases de dados, estudos que não atendiam o recorte temporal ou que estavam publicados em outros idiomas.

Após a filtragem dos dados, com base nos critérios de inclusão e exclusão, foram elencados 4 artigos para o estudo. Os artigos passaram por uma leitura e análise minuciosa, onde foram constatados dois estudos teóricos, que visavam elencar os sintomas e causas da SB, e dois estudos práticos, que além de analisar sintomas e causas, aplicaram os conhecimentos com profissionais de Educação Física. A síntese foi elaborada na discussão deste artigo.

\section{RESULTADOS}

A busca inicial apontou 580 artigos, dos quais 100 foram excluídos por motivo de duplicação, outros 100 foram excluídos após a leitura dos resumos, 100 foram excluídos devido ao tempo de publicação, 200 foram excluídos após uma leitura minuciosa, 76 foram excluídos por serem pagos, sendo que foram restantes quatro artigos que se enquadraram nos critérios de inclusão e exclusão, conforme a tabela 1. Para a análise dos resultados, os seguintes dados foram extraídos: Educação Física; Síndrome de Burnout; Transtornos Mentais.

Apesar do ano de publicação ser restrito aos 10 anos anteriores, optou-se por utilizar artigos primários sobre o tema, os quais são de grande relevância para o estudo atualmente abordado. 


\section{artigo}

Oliveira, R.F.P.; Pereira, M.O.; Gomes Junior, E.S.; Oliveira, R.C.; Pontini, A.M.; Rosário, K.R.;

Contribuições da religiosidade no enfrentamento da sindrome de burnout em profissionais de educação física

\section{Tabela 1: Objetivo e conclusão dos 04 artigos incluídos na pesquisa.}

\begin{tabular}{|c|c|c|c|c|}
\hline & AUTOR & TÍTULO & BASE DE DADOS/ANO & OBJETIVOS \\
\hline 1 & $\begin{array}{l}\text { SILVA, S. C.P.S., } \\
\text { et al. }\end{array}$ & $\begin{array}{c}\text { A Síndrome de Burnout em } \\
\text { profissionais da Rede de Atenção } \\
\text { Primária à Saúde de Aracaju, } \\
\text { Brasil. }\end{array}$ & $\begin{array}{l}\text { Scientific Eletronic Library } \\
\text { On-line (SciELO)/ } 2015 .\end{array}$ & $\begin{array}{c}\text { Avaliar a prevalência da SB e fatores associados } \\
\text { em profissionais de nível superior vinculados à } \\
\text { Rede de Atenção Primária à Saúde do município } \\
\text { de Aracaju/SE. }\end{array}$ \\
\hline 2 & DIEHL, L., et al. & $\begin{array}{l}\text { Adoecimento mental em } \\
\text { professores brasileiros: revisão } \\
\text { sistemática da literatura }\end{array}$ & $\begin{array}{l}\text { Portal de periódicos da } \\
\text { CAPES }\end{array}$ & $\begin{array}{l}\text { Identificar principais sintomas e/ou adoecimen- } \\
\text { tos psíquicos entre professores brasileiros. }\end{array}$ \\
\hline 3 & $\begin{array}{l}\text { CACCIARI, M, B., } \\
\text { et al. }\end{array}$ & $\begin{array}{l}\text { Percepções de professores } \\
\text { universitários brasileiros sobre } \\
\text { as virtudes mais valorizadas no } \\
\text { exercício da docência }\end{array}$ & $\begin{array}{l}\text { Scientific Eletronic Library } \\
\text { On-line (SciELO)/ } 2017 .\end{array}$ & $\begin{array}{c}\text { Investigar as virtudes necessárias para ser um } \\
\text { bom professor e o quanto os participantes per- } \\
\text { cebe que tais características os descrevem como } \\
\text { profissionais. }\end{array}$ \\
\hline 4 & $\begin{array}{l}\text { SILVA, J. L. L., } \\
\text { et al. }\end{array}$ & $\begin{array}{l}\text { Prevalência da síndrome de Bur- } \\
\text { nout entre professores da Escola } \\
\text { Estadual em Niterói, Brasil. }\end{array}$ & $\begin{array}{l}\text { Portal de periódicos da } \\
\text { CAPES }\end{array}$ & $\begin{array}{c}\text { O objetivo deste estudo foi descrever a prevalên- } \\
\text { cia da Síndrome de Burnout entre os professores } \\
\text { da Escola Estadual em Niterói, Brasil. }\end{array}$ \\
\hline
\end{tabular}

Tabela 2: Descrição dos artigos inclusos na pesquisa, sendo o autor, metodologia e conclusão.

AUTOR

TIPO DE ESTUDO

1 SILVA, S. C.P. S., et al

Pesquisa quantitativa.

Revisão Sistemática da Literatura Nacional.

3

CACCIARI, M, B.,
et al.

4
SILVA, J. L. L., et al.
Estudo quantitativo e descritivo.

\section{CONCLUSÃO}

Esses achados sugerem a importância da implantação de medidas preventivas e interventivas voltadas a esses profissionais, de forma a garantir uma melhoria no ambiente de trabalho.

Espera-se que esses resultados direcionem novas pesquisas e estimulem o planejamento de intervenções eficazes voltadas para a saúde mental de professores.

De acordo com os resultados obtidos, as virtudes mais valorizadas no exercício docente foram conhecimento, temperança, justiça e humanidade. Estes resultados ajudam a pensar nas habilidades e competências exigidas na vida acadêmica, assim como na avaliação das práticas docentes.

Conclui-se que, a partir dos dados analisados, observou-se que a profissão não possui intervenções para melhorar a qualidade de vida dos professores em seus trabalhos.

\section{DISCUSSÃo}

A religiosidade é a revelação do sagrado onde essa força sobrenatural apresenta o poder ${ }^{(7)}$. Igualmente, caracteriza-se enquanto um sistema organizado, no qual as práticas, rituais, crenças e símbolos são utilizados com a finalidade de aproximação ao transcendente ${ }^{(6,7,8)}$. Para tanto, percebe-se que esta representa um elemento social fundamental, uma vez que, são desenvolvidos os valores que formam o homem e a sociedade. Isto é, através dela manifesta-se o sagrado conjuntamente com a construção de preceitos fundantes do indivíduo, da família e da comunidade ${ }^{(6)}$.

Ainda, a religiosidade é considerada uma representação dos aspectos referentes à moral e a ética do indivíduo. Portanto, alcança a dimensão da subjetividade no que tange às escolhas relativas à fé. Para isso, compreende-se que ela transcende a materialidade, em que o sentido de vida está no equilíbrio das esferas racional, afetiva e social ${ }^{(7)}$.

Dito isto, a religiosidade, entendida como uma forma particular de espiritualidade, juntamente, mostrou-se eficiente no enfrentamento de enfermidades em pacientes com depressão e problemas de saúde mental ${ }^{(6)}$. A oração pode ser definida de várias maneiras, com base em crenças individuais e tradições religiosas, mas a oração pessoal envolve, sobretudo, elevar os pensamentos em Deus. De tal modo, a oração tem sido considerada como um poderoso mecanismo espiritual de enfrentamento das doenças, e a percepção do transcendente teve uma influência positiva ante o comprometimento da saúde relacionado ao estresse ${ }^{(7)}$.

No tocante à área educacional, geralmente o ritmo de trabalho é intenso e são exigidos altos níveis de atenção e concentração para a realização das tarefas e na escola, o trabalho mais diretamente vinculado à produção, é o realizado pelo educador, daí a maior pressão do sistema incidir sobre o docente ${ }^{(9)}$.

A profissão de Educação Física, ou qualquer outra, pode gerar prazer, como também 
insatisfação, quando gerado prazer, a pessoa se encontra em plena paz consigo e com os outros. Entretanto, quando gerado insatisfação com aquilo que está exercendo, a pessoa se encontra irritada ou estressada e se a insatisfação for preponderante os níveis de estresse tenderão a subir e as consequências terão reflexo em todo o sistema ${ }^{(10,11,12,13)}$.

Nesse sentido, fatores como sobrecarga de trabalho, pressão de tempo e ambiente organizacional desfavorável, são algumas das fontes de estresse que estes profissionais mais descrevem e que estão relacionadas ao seu esgotamento emocional ${ }^{(6)}$. No entanto, existem outras causas que parecem estar associadas também ao esgotamento: instabilidade no trabalho, pouca promoção e mobilidade social, baixos incentivos econômicos, falta de recursos materiais e pessoais nos centros, estilos de supervisão caracterizados por pouco apoio e numerosas atitudes negativas em relação aos profissionais $(5,6,9,10,11)$.

A profissão de educador acaba sendo, então, uma das mais estressantes da atualidade, e a causa mais frequente que levam estes profissionais a sofrerem com a Síndrome de Burnout (SB), é a falta de estrutura dos ambientes de trabalho. Pois, a temperatura, muitas vezes é elevada nas épocas mais quentes, a iluminação inadequada e o barulho intenso, acabam prejudicando o trabalho do professor, e distraindo os alunos, fazendo com que eles não prestem atenção na aula ${ }^{(9,10,11)}$.

No que se refere à relação entre saúde e religiosidade, observa-se, historicamente, que ela se confunde com a própria narrativa da humanidade. Destarte, verifica-se que as comunidades religiosas foram as responsáveis pela criação de casas de apoio à saúde, tanto espiritual quanto física e mental. Destacando-se, através da consagração da fé, as atitudes que promoveram benefícios no enfrentamento de condições negativas relacionadas à saúde mental ${ }^{(7)}$.

\section{CONCLUSÃO}

A prevenção de doenças em profissionais de Educação Física não é tarefa solitária, mas existe uma ação coletiva, de modo que as reflexões e discussões geradas devem visar à busca de alternativas para possíveis modificações. Trata-se assim de religar estes profissionais à tradição que se encontra na raiz política de sua vocação, educador é aquele que professa e ensina uma ciência, uma arte, uma técnica, uma disciplina é um mestre e é um perito. É também aquele que professa publicamente sua crença numa verdade, remeter os professores ao seu nome significa afirmar acima de tudo o direito à transmissão, com todas suas exigências internas, é certo, mas com todos os seus efeitos imponderáveis, contra todas as manobras que colocam o futuro do educando.

Esse artigo contribui para o conhecimento do processo da Síndrome de Burnout na atividade do profissional de Educação Física, proporcionando o despertar para elaboração de programas de prevenção do estresse e resultando, dessa forma, na conquista de uma vida mais saudável, tanto na área física quanto psicológica. Igualmente, o reconhecimento da religiosidade, quando harmonizada, enquanto elemento capaz de enfrentar os sintomas provocados pelo estresse, uma vez que, o envolvimento religioso apresenta resultado positivo ante a saúde mental, Síndrome de Burnout.

Em conclusão, a Síndrome de Burnout ocorre, devido à sobrecarga exigida dos profissionais de Educação Física, de modo que os futuros profissionais devem saber lidar com todas as exigências e responsabilidades futuras, assim como saber equilibrar tais funções dentro da sua área de atuação.

\section{REFERÊNCIAS}

1. Brasil. Classificação Brasileira de Ocupações. Ministério do Trabalho. 2020.

2. Costa VT, Albuquerque MR, Samulsk DM. A síndrome do Burnout em profissionais de Educação Física: perspectivas e desafios. EFDEPORTES Revista Digital. 2011; 16(159):1-5.

3. Castro AG. A invenção do trabalhismo. São Paulo: Cortez. 2015.

4. Dias KM, Campos LA, Freitas MR, Ferreira AA. Prevalência da síndrome de Burnout em professores do curso de medicina de uma faculdade particular de Belo Horizonte. Revista Interdisciplinar Ciências Médicas. 2019; 3(2):42-7.

5. Maslach C, Jackson SE. The measurement of experienced burnout. J Ocup Behav. 2013; 2:99-113.

6. Zanetti G, et al. Percepção de Acadêmicos de Medicina e de Outras áreas da Saúde e Humanas (Ligadas à Saúde) sobre as Relações entre Espiritualidade, Religiosidade e Saúde. Revista Brasileira de Educação Médica. 2018; 42(1):62-72.

7. Cerqueira D. Religião, religiosidade e contexto do trabalho. So- ciedade e Estado. 2005; 20(3):717-24.

8. Bernardi CJ, Castilho MA. A religiosidade como elemento do desenvolvimento humano. Interações. 2016; 17(4):745-56.

9. França $A C$, Rodrigues AL. Stress e trabalho: uma abordagem psicossomática. 5 ed. São Paulo: Atlas. 2008.

10. Ramón JPM. Cómo se defiende el profesorado de secundaria del estrés: Burnout y estrategias de afrontamiento. Revista de Psicología del Trabajo y de las Organizaciones. 2015; 13(1):1-9.

11. Carlotto MS, et al. O papel mediador da auto eficácia na relação entre a sobrecarga de trabalho e as dimensões de Burnout em professores. Psico-USF. 2015; 20(1):13-23.

12. Frick W, et al. Fatalities at wind turbines may threaten population viability of a migratory bat. Biological Conservation. 2017; 209:172-7.

13. Pêgo FPL, et al. Síndrome de Burnout. Revista Brasileira de Medicina do Trabalho. 2016; 14(2):171-6. 